\title{
CORRECTION
}

Yi Zhang $\cdot$ Chenxi Yu $\cdot$ Ruoqi Wang $\cdot$ Xunhan Liu

\section{Correction to: Visual dimension analysis based on dimension subdivision}

Published online: 9 April 2021

(C) The Visualization Society of Japan 2021

Correction to: J Vis (2021) 24:117-131 https://doi.org/10.1007/s12650-020-00694-3

Unfortunately, the acknowledgment was not included in the online published article. Now, the acknowledgment is given below.

"The paper "Visual dimension analysis based on dimension subdivision" is supported by National Natural Science Foundation of China (61702359)".

The original article can be found online at https://doi.org/10.1007/s12650-020-00694-3.

Y. Zhang $(\bowtie) \cdot$ C. Yu $\cdot$ R. Wang $\cdot$ X. Liu

College of Intelligence and Computing, Tianjin University, Tianjin, China

E-mail: yizhang@tju.edu.cn

C. $\mathrm{Yu}$

E-mail: yuchenxi@tju.edu.cn

R. Wang

E-mail: wangrq07@163.com

X. Liu

E-mail: xunhanliu@outlook.com 